\title{
Mortality in hypoplastic left heart syndrome: Review of 216 autopsy cases of aortic atresia with attention to coronary artery disease
}

\author{
Meena Nathan, MD, ${ }^{\mathrm{a}}$ Alex K. Williamson, MD, ${ }^{\mathrm{b}}$ John E. Mayer, MD, ${ }^{\mathrm{a}}$ Emile A. Bacha, $\mathrm{MD},{ }^{\mathrm{c}}$ and \\ Amy L. Juraszek, MD ${ }^{\mathrm{d}}$
}

\begin{abstract}
Objectives: Aortic atresia (AA) in hypoplastic left heart syndrome (HLHS) has been associated with increased mortality in several prior studies. We reviewed our autopsy series to explore the relationship of coronary abnormalities to anatomic subsets of HLHS with AA.
\end{abstract}

\begin{abstract}
Methods: We retrospectively reviewed all pathology specimens with AA/MS (mitral stenosis) and AA/MA (mitral atresia) in the Cardiac Registry of Children's Hospital Boston between 1955 and 2009 including autopsy reports, operative notes, and imaging studies. Formalin-fixed hearts were examined, and cases found to have macroscopic coronary artery abnormalities were sectioned at mid-left ventricular level in the transverse plane and at mid-right ventricular level in the longitudinal plane for histologic analysis of coronary arteries using tissue sections stained with hematoxylin and eosin.
\end{abstract}

Results: A total of 216 autopsy cases were identified with AA/MS (134) and AA/MA (82). Coronary anomalies were found in 49 cases, left ventricle-coronary fistula in 39, all in AA/MS, and 10 other coronary abnormalities, all in AA/MA. Histologic study confirmed fistulas only in the AA/MS group with no evidence of fistulas in the AA/MA group.

Conclusions: The occurrence of left ventricle-coronary fistulas appears limited to the AA/MS group, and coronary fistula specimens were disproportionately more prevalent in postoperative specimens. Further clinical studies are required to validate this finding and to identify subgroups that carry a higher mortality risk. (J Thorac Cardiovasc Surg 2012;144:1301-6)

Prior reports on hypoplastic left heart syndrome (HLHS) at our and other institutions have found that the aortic atresia/ mitral stenosis (AA/MS) subgroup was associated with a higher mortality. Vida and associates ${ }^{1}$ reported a higher $(29 \%$ vs $7.9 \%)$ mortality in the AA/MS variant of HLHS compared with other anatomic subtypes of HLHS. Glatz and colleagues ${ }^{2}$ reported a $21 \%$ vs $3 \%$ mortality in the AA/MS subgroup vs the aortic atresia/mitral atresia (AA/MA) subgroup.

The pathophysiologic mechanisms underlying these findings have not been clearly defined, but a relationship to coronary abnormalities has been postulated. Several coronary abnormalities have been documented in HLHS, with the most common being coronary fistulas or ventricular-

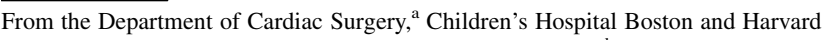
Medical School, Boston, Mass; the Division of Pathology, ${ }^{b}$ Long Island Jewish/ North Shore Hospital, Cohen Children's Hospital, and Hofstra Medical School, New Hyde Park, NY; the Division of Congenital and Pediatric Heart Surgery, ${ }^{\mathrm{c}}$ Morgan Stanley Children's Hospital of New York, Columbia Presbyterian University, New York, NY; and the Division of Pediatric Cardiology, ${ }^{\mathrm{d}}$ UT Southwestern Medical Center, Children's Medical Center, Dallas, Tex.

Disclosures: Authors have nothing to disclose with regard to commercial support. Received for publication July 29, 2011; revisions received Jan 22, 2012; accepted for publication March 12, 2012; available ahead of print April 12, 2012.

Address for reprints: Meena Nathan, MD, Department of Cardiac Surgery, Children's Hospital Boston, Bader 273, 300 Longwood Ave, Boston, MA 02115 (E-mail: meena.nathan@cardio.chboston.org).

$0022-5223 / \$ 36.00$

Copyright (C) 2012 by The American Association for Thoracic Surgery doi:10.1016/j.jtcvs.2012.03.013
}

coronary connections. O'Connor and coworkers ${ }^{3}$ in 1982 found that the AA/MS subgroup had a greater number of coronary abnormalities including ventricular-coronary connections. Baffa and associates ${ }^{4}$ reviewed 151 autopsy specimens, 89 with AA/MS and 52 with AA/MA. They identified 27 coronary-cameral fistulas in the AA/MS subgroup, 17 with associated tortuous epicardial vessels, and 3 specimens had single coronaries. Other abnormalities that have been described include intramural coronaries, high origin of coronaries, and anomalous origin of the left coronary artery from the pulmonary artery.

We hypothesized that the occurrence of ventricular-coronary fistula would be more common in the AA/MS subgroup because of the frequent occurrence of hypertensive left ventricles (LVs) in this group. Ventricular-coronary fistulas could contribute to coronary steal and myocardial dysfunction and may contribute to mortality in this subgroup. To better understand the relationship between coronary disease and mortality, we undertook a review of all autopsies performed on patients with HLHS and AA at our center.

\section{METHODS}

We retrospectively reviewed, with institutional review board approval, all cases of AA subtype of HLHS that underwent autopsy in the Cardiac Registry of Children's Hospital Boston between 1955 and 2009. Clinical charts were reviewed for clinical history and operative data. Reports of all preoperative and postoperative imaging studies and autopsies were 


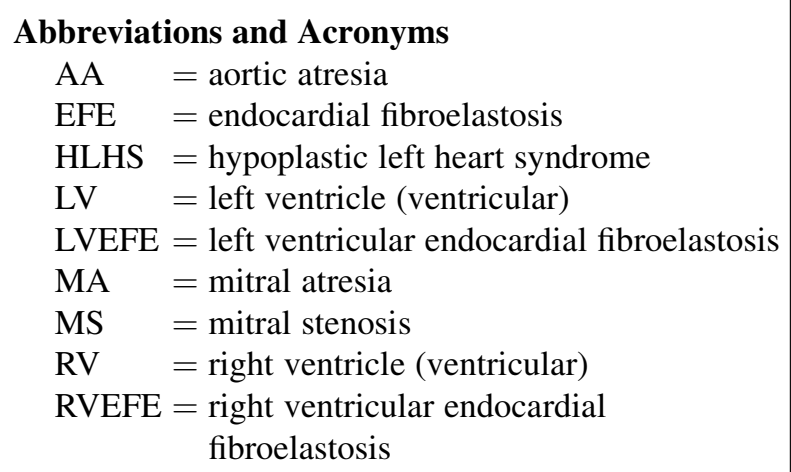

reviewed. In addition, all available formalin-fixed HLHS/AA heart specimens were examined, including gross examination of coronaries in all hearts.

MS was defined by documented flow across the mitral valve in imaging studies or by the presence of a well-defined mitral valve with a patent inflow into the LV and a measurable orifice.

Histologic examination of all AA hearts with coronary abnormalities noted on echocardiography, cardiac catheterization, or on gross autopsy examination was performed. In addition, coronary histologic examination was also carried out on an equivalent number of AA hearts matched for age and surgical status. Two representative tissue blocks, the first a cross section of the LV at mid cavity including the septum and the second a longitudinal section from the right ventricle (RV) at the acute margin, including the atrioventricular sulcus, were taken from intact heart specimens that had been fixed in $10 \%$ buffered formalin. The tissue was embedded in paraffin, and for each block, 5- $\mu$ m-thick sections were cut and stained with hematoxylin and eosin and examined with an Olympus BX400 microscope (Olympus Corporation, Tokyo, Japan) with field diameters calibrated using a stage micrometer. Measurements were made of (1) thicknesses of the ventricular wall, (2) thickness of the medial layer of coronary arteries having an outer diameter between $1100 \mu \mathrm{m}$ and $2200 \mu \mathrm{m}$, and (3) thickness of any ventricular fibroelastosis approximated to the nearest fraction $(1 / 6,1 / 4,1 / 3$, $1 / 2,2 / 3$, or 3/4) of the microscopic field in which the thickness of interest was completely visualized (eg, fibroelastosis that spanned half of a 2200$\mu \mathrm{m}$-wide microscopic field was determined to have a thickness of 1100 $\mu \mathrm{m})$. A fistula between a coronary artery and a ventricular cavity was considered present if there was an unambiguous connection on microscopic examination or if there was overlap of a coronary artery and a ventricular cavity in the plane of the slide. In histologically ambiguous cases or for cases in which fistulas were grossly identified but not seen in the initial hematoxylin and eosin slide, additional hematoxylin and eosin slides were prepared from the respective blocks at $10-\mu \mathrm{m}$ intervals and examined as above.

The hearts were classified into 4 groups:

- Group 1: AA/MS (aortic atresia, mitral stenosis) — hearts with a diagnosis of AA/MS confirmed by gross examination.

- Group 2: AA/MS/CF (aortic atresia, mitral stenosis, coronary fistula) hearts in the AA/MS group that had coronary abnormalities on echocardiography, cardiac catheterization, gross examination, or histologic examination.

- Group 3: AA/MA (aortic atresia, mitral atresia)—hearts with diagnosis of AA/MA confirmed by gross examination.

- Group 4: AA/MA/CA (aortic atresia, mitral atresia, coronary abnormalities) - hearts in the AA/MA group that had coronary abnormalities on echocardiography, cardiac catheterization, gross examination, or histologic examination.
This grouping system was used for comparison between groups and for statistical analysis of histologic findings.

A nonparametric test (Mann-Whitney) was used to compare the median coronary artery medial thickness and thickness of LV endocardial fibroelastosis (LVEFE) among the 4 groups. The Pearson $\chi^{2}$ test was used to compare the proportions of 4 groups in the pre-Norwood (preoperative) and post-Norwood (palliative surgical) eras. The statistics package used for analysis was PASW Statistics 18.0, SPSS Inc, Chicago, Ill.

\section{RESULTS}

A total of 216 hearts with the diagnosis of HLHS and AA were examined at the cardiac registry at Children's Hospital Boston between 1955 and 2009. Of these, 200 were autopsy specimens, and 16 were hearts explanted at orthotopic heart transplantation. All but 2 of the 216 hearts were available for reexamination. One hundred thirty-four $(62 \%)$ were of the AA/MS subtype and the remaining $82(38 \%)$ were of the AA/MA subtype. Thirty-nine $(29 \%)$ of 134 specimens of the AA/MS subtype had coronary abnormalities, and all were fistulas between the ventricle and coronary arteries, with $38 \mathrm{LV}$-coronary fistulas and $1 \mathrm{RV}$-coronary fistula. Figure $1, A$ and $B$, shows representative cardiac catheterization images of LV-coronary fistulas. In the AA/MA group, 10 specimens had coronary abnormalities $(12 \%)$, but none had coronary fistulas. The abnormalities noted in the AA/MA group included anomalous origin of the left coronary artery from the pulmonary artery in 1 , single coronary artery in 3 ( 1 with an atretic left coronary ostium), aneurysmal right coronary artery in 1 , high takeoff and intramural right coronary artery in 1, intramyocardial left anterior descending coronary artery in 1, coronary ostial stenosis in 1, hypoplastic coronaries in 1, and high takeoff and oblique ostium of left coronary artery in 1 .

A total of 96 hearts were examined histologically: 31 in $\mathrm{AA} / \mathrm{MS}, 37$ in $\mathrm{AA} / \mathrm{MS} / \mathrm{CF}, 16$ in AA/MA, and 10 in AA/ MA/CA groups. Figure 2, $A$ and $B$, shows histologic images of coronary fistulas.

Tables 1 and 2 give an outline of management strategies that had been used before the time of death/explant, and management stage at death/explant, in the 4 anatomic subgroups. Tables 3 and 4 document coronary medial thickness and EFE thickness in the 4 groups.

On comparison of the 4 groups on the basis of historical era, ventricular-coronary fistulas were found much more frequently in the AA/MS group in the postoperative era than in the era before first attempts at surgical palliation $(P<.031)$. This finding of a higher percentage of autopsied hearts in the operated groups compared with the nonoperated specimens suggests an unfavorable impact of ventricular-coronary fistulas on surgical palliative procedures. The presence of LVEFE was significantly higher $(P>.0001)$ in the $\mathrm{AA} / \mathrm{MS}$ and $\mathrm{AA} / \mathrm{MS} / \mathrm{CF}$ groups than in the $\mathrm{AA} / \mathrm{MA}$ and AA/MA/CA groups. However, there was no significant difference between the AA/MS and the AA/MS/CF groups in thickness of the LVEFE. Interestingly, there was a higher 

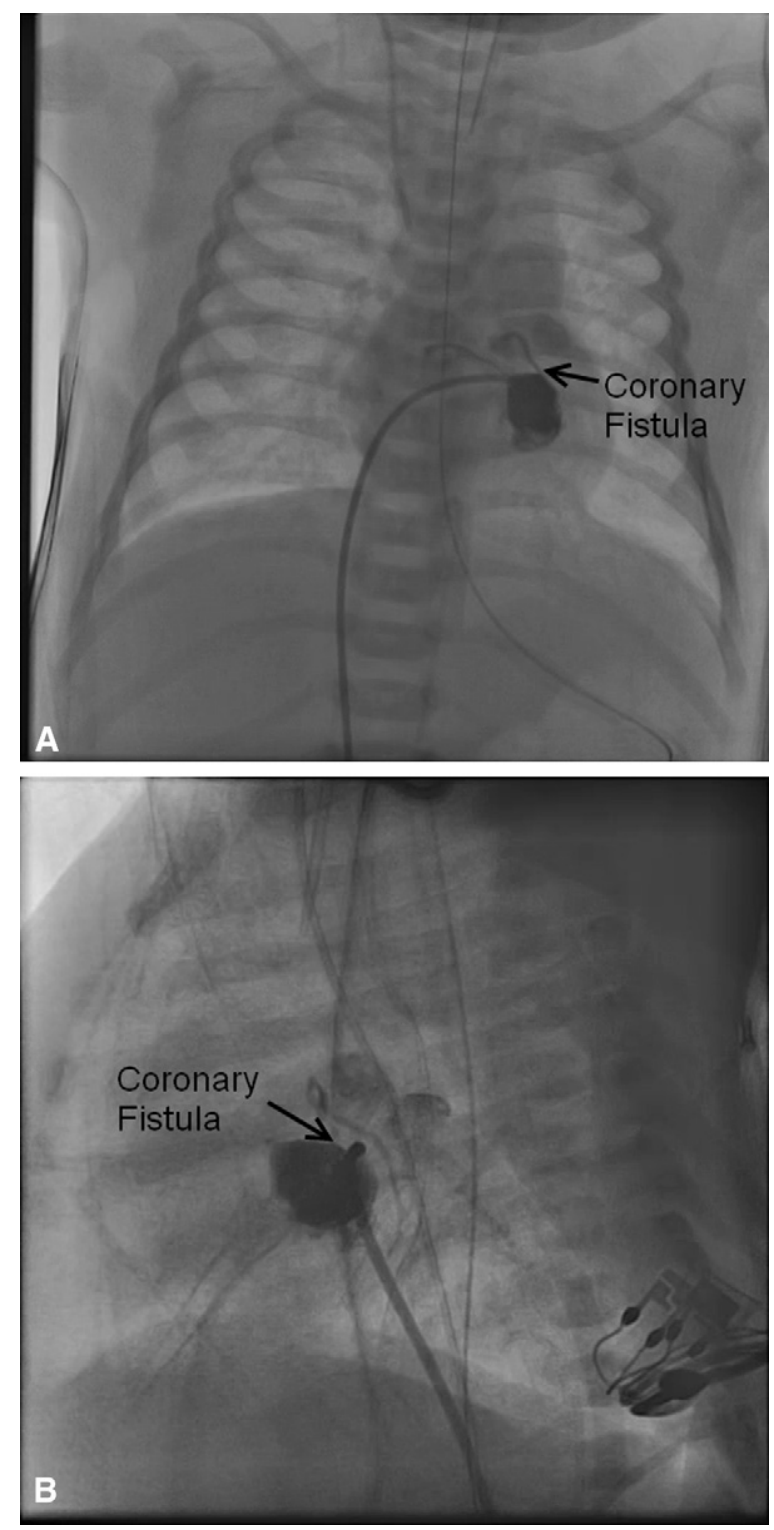

FIGURE 1. A, Anteroposterior view of a left ventricular angiogram demonstrating retrograde filling of the left coronary artery via the fistula. Arrow points to the fistula. B, Lateral view of the same left ventricular angiogram demonstrating the coronary fistula filling the left coronary artery on injecting the left ventricle. Arrow points to the fistula.

occurrence of RV endocardial fibroelastosis (RVEFE) in the $\mathrm{AA} / \mathrm{MS} / \mathrm{CF}$ group than in the AA/MS group $(P<.030)$.

\section{DISCUSSION}

Since first description of the pathophysiology of HLHS by Bardeleben in $1851,{ }^{6}$ this disease complex has been extensively studied. HLHS was a uniformly fatal condition until palliative procedures were developed and perfected in the 1970s and early 1980s. In 1981 Norwood and coworkers ${ }^{7}$ reported their experience with 16 consecutive infants with HLHS in whom staged palliation was attempted. Of interest,
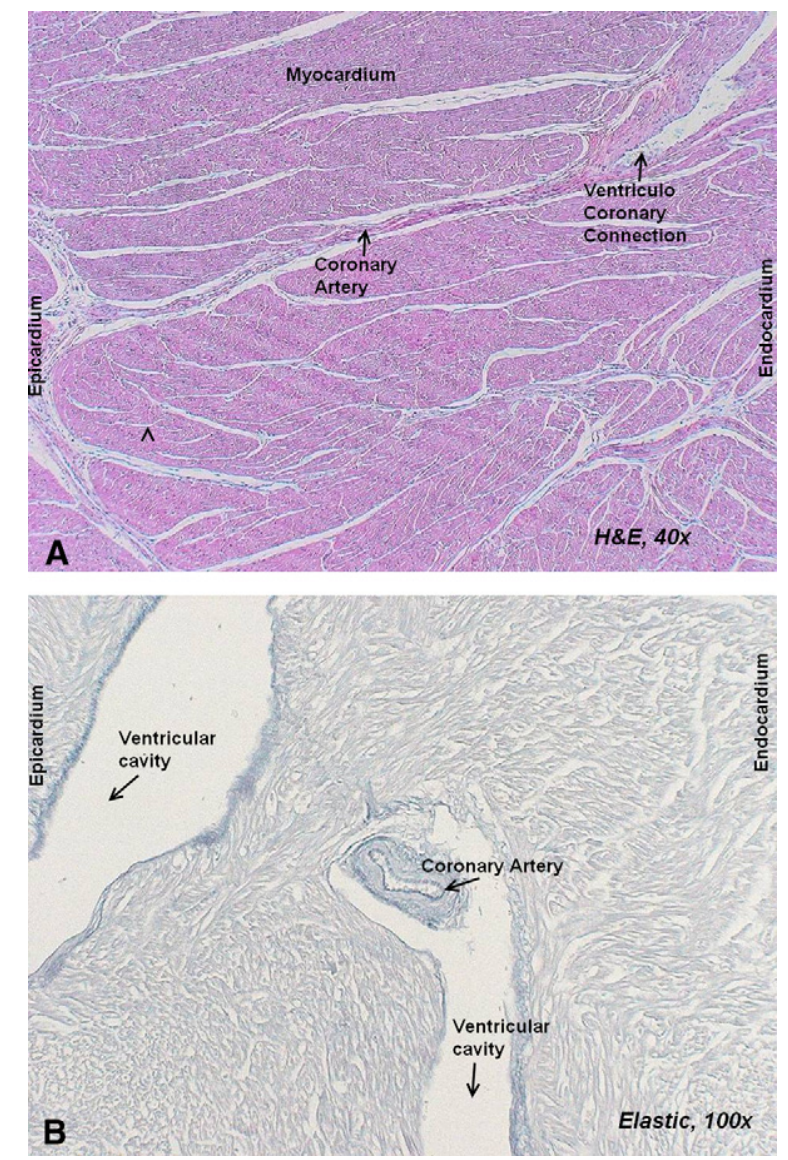

FIGURE 2. A, Histologic section of the left ventricle stained with hematoxylin and eosin $(H \& E)$ at $40 \times$ magnification. Coronary artery and ventricular-coronary connections are marked by solid black arrows. B, Elastic-stained histologic section of the left ventricle at $100 \times$ magnification. The coronary artery opening into the ventricular cavity is marked by solid arrows.

3 of these patients had LV-left anterior descending coronary artery fistulas, which are included in our series. Our series includes specimens obtained after a variety of palliative procedures, including the first Norwood procedures.

\section{Clinical and Pathologic Distribution of the AA/MS and AA/MA Subtypes}

In contrast to the current pathologic series, most clinical series of HLHS with AA have had a higher occurrence of AA/MA subtype. Mahle and his group ${ }^{8}$ in their series of 840 HLHS patients found 520 patients with AA; $61 \%$ of this group had AA/MA whereas $39 \%$ had AA/MS. A similar distribution of $65 \%$ AA/MA vs $35 \%$ AA/MS was reported by Glatz and colleagues. ${ }^{2}$

Some early autopsy series have also reported a similar distribution. Van Praagh and associates, ${ }^{9}$ in their review of nearly 3000 hearts in the cardiac registry at Children's Hospital Boston, reported 102 cases of HLHS with AA with $63 \%$ in the AA/MA subgroup and $37 \%$ in the 
TABLE 1. AA management strategy

\begin{tabular}{lcccc}
\hline \multicolumn{1}{c}{ Management } & $\begin{array}{c}\text { AA/MS } \\
(\mathbf{n}=\mathbf{1 3 4})\end{array}$ & $\begin{array}{c}\mathbf{A A} / \mathbf{M S} / \mathbf{C F} \\
(\mathbf{n}=\mathbf{3 9})\end{array}$ & $\begin{array}{c}\text { AA/MA } \\
(\mathbf{n = 8 2})\end{array}$ & $\begin{array}{c}\text { AA/MA/CA } \\
(\mathbf{n}=\mathbf{1 0})\end{array}$ \\
\hline $\begin{array}{l}\text { I no surgery } \\
\text { (before Norwood era) }\end{array}$ & $49 *$ & $8 *$ & 32 & 4 \\
$\begin{array}{l}\text { II surgical palliation } \\
P \text { value }\end{array}$ & $85 \dagger$ & $\begin{array}{c}31 \dagger \\
\text { (I vs II) }\end{array}$ & 50 & 6 \\
\hline
\end{tabular}

This table represents the distribution of the hypoplastic left hearts with aortic atresia in the 3 subgroups in the preoperative and postoperative eras. There is a significantly higher proportion of hearts with AA/MS and ventricular-coronary fistulas in the postoperative era. $A A$, Aortic atresia; $M S$, mitral stenosis; $C F$, coronary fistula; $M A$, mitral atresia; $C A$, coronary anomalies other than coronary fistula; $N S$, not significant. *Explanted heart from primary orthotopic heart transplantation $=1$. $\dagger$ Hybrid stage $\mathrm{I}=4$.

AA/MS subgroup. This finding is likely related to the fact that the majority of cases reported in this series were from the era before attempts at surgical palliation. However, later autopsy series have contained a higher percentage of AA/ MS hearts. Bartram and colleagues ${ }^{10}$ reported 88 cases of AA with a distribution of $49 \%$ AA/MA and $51 \%$ AA/MS in their postmortem series of 122 deaths after modified Norwood procedures. Bharati and Lev ${ }^{11}$ looked at 230 hearts with HLHS. They reported 200 cases of AA, $95(48 \%)$ with AA/MA, and $105(52 \%)$ with AA/MS.

In our series of 216 autopsy cases of HLHS/AA, we found a much higher percentage of specimens with AA/ MS compared with AA/MA ( $62 \%$ vs $38 \%$ ). This finding is potentially related to the significantly higher number of coronary abnormalities seen in the AA/MS group, $29 \%$ vs $12 \%$ in the AA/MA group $(P<.004)$ and to the inclusion of a large number $(63 \%)$ of postoperative specimens. The only coronary abnormality we found in the AA/MS subgroup was ventricular-coronary fistula. The AA/MA group had several types of coronary abnormalities but none had coronary fistulas.

\section{Coronary Morphology}

Prior studies of the coronary arteries in HLHS suggested that coronary morphology was a possible contributor to

TABLE 2. AA stage at death

\begin{tabular}{lcccc}
\hline \multicolumn{1}{c}{ Outcome } & $\begin{array}{c}\text { AA/MS } \\
(\mathbf{n}=\mathbf{1 3 4})\end{array}$ & $\begin{array}{c}\text { AA/MS/CF } \\
(\mathbf{n}=\mathbf{3 9})\end{array}$ & $\begin{array}{c}\text { AA/MA } \\
(\mathbf{n}=\mathbf{8 2})\end{array}$ & $\begin{array}{c}\text { AA/MA/CA } \\
(\mathbf{n}=\mathbf{1 0})\end{array}$ \\
\hline $\begin{array}{l}\text { Unoperated } \\
\quad \text { before Norwood era) }\end{array}$ & $49^{*}$ & $8^{*}$ & 32 & 4 \\
Stage I & 59 & 20 & 30 & 5 \\
OHT after stage I & 8 & 7 & 0 & 0 \\
Interstage death & 4 & 0 & 13 & 1 \\
After BDG death & 4 & 2 & 3 & 0 \\
OHT after BDG & 0 & 0 & 3 & 0 \\
After Fontan death & 6 & 0 & 1 & 0 \\
OHT after Fontan & 4 & 2 & 0 & 0 \\
\hline
\end{tabular}

This table represents the proportion of hearts in the 4 subgroups based on stage at death. Again there is higher proportion of hearts with AA/MS and ventricular-coronary fistula in the postoperative era, particularly after the initial palliation. $A A$, Aortic atresia; $M S$, mitral stenosis; $C F$, coronary fistula; $M A$, mitral atresia; $C A$, coronary anomalies other than coronary fistula; $O H T$, orthotopic heart transplantation; $B D G$, bidirectional Glenn. *Explanted heart after orthotopic heart transplantation.
TABLE 3. Histology of coronary arteries-medial thickness

\begin{tabular}{lcccc}
\hline \multicolumn{5}{c}{ Coronary artery medial thickness $(\boldsymbol{\mu m})$} \\
\hline & AA/MS & AA/MS/CF & AA/MA & AA/MA/CA \\
\hline $\mathrm{n}$ & 31 & 37 & 16 & 10 \\
Median & 37 & 55 & 37 & 37 \\
Range & $37-165$ & $37-110$ & $37-110$ & $37-110$ \\
$P$ value & \multicolumn{5}{c}{. } \\
\hline
\end{tabular}

This table compares the coronary artery medial thickness in the 4 groups of hypoplastic left hearts with aortic atresia. There is no significant difference in the medial thickness of coronary arteries noted. $A A$, Aortic atresia; $M S$, mitral stenosis; $C F$, coronary fistula; $M A$, mitral atresia; $C A$, coronary anomalies other than coronary fistula.

mortality. Lloyd, with Evans and Marvin ${ }^{12}$ and with Marvin, ${ }^{13}$ compared 51 autopsy specimens with HLHS with 18 normal controls and found no difference in coronary ostial size or diameters in the 2 groups. A subsequent study of 59 HLHS heart specimens, all from patients who died within the first 90 days after birth (11 surgically palliated), demonstrated a significant survival advantage for larger coronaries, greater RV mass, and coronary/RV mass ratio. They concluded that adequacy of myocardial perfusion was essential for survival. Sauer and colleagues ${ }^{14}$ compared the epicardial coronaries in $9 \mathrm{AA} / \mathrm{MA}$ hearts, $19 \mathrm{AA} / \mathrm{MS}$ hearts, and 10 normal hearts. They noted that LVEFE was present in all AA/MS hearts and that coronaries in AA/ MS hearts were thicker and more tortuous on macroscopic examination. Although there was no difference in external diameters of the coronaries in the groups, some AA/MS hearts did show an increase in medial thickness, particularly in the left coronary system. They hypothesized that these coronary abnormalities may increase risk of mortality.

Baffa and coworkers, ${ }^{4}$ in their series of 151 hearts, reported no difference in coronary artery wall thickness between AA/MS, AA/MA, and AS/MS hearts. Sugiyama and associates ${ }^{15}$ described a higher occurrence of abnormal findings, including myocardial necrosis, interstitial fibrosis, and calcification, in the MS subgroups of HLHS, which could possibly contribute to RV dysfunction and thus poorer prognosis. Recent studies by Salih, Sheppard, and $\mathrm{Ho}^{16}$ on unoperated HLHS hearts from patients dying in the first 2

TABLE 4. Histology of LV endocardium

\begin{tabular}{lcccc}
\hline \multicolumn{5}{c}{ EFE thickness $(\boldsymbol{\mu m})$} \\
\hline $\mathrm{n}$ & AA/MS & AA/MS/CF & AA/MA & AA/MA/CA \\
Median & 31 & 37 & 16 & 10 \\
Range & 225 & 225 & 0 & 0 \\
$P$ value & $0-1833$ & $0-1833$ & $0-73$ & $0-73$ \\
& $<.0001 \mathrm{vs}$ & $<.0001 \mathrm{vs}$ & & \\
& AA/MA & AA/MA/CA & & \\
\hline
\end{tabular}

This table compares the thickness of the left ventricular endocardium in the four groups of hypoplastic left hearts with aortic atresia. There is a significant difference in the occurrence of left ventricular fibroelastosis in the subgroup with aortic atresia and mitral stenosis. $L V$, Left ventricle (ventricular); $E F E$, endocardial fibroelastosis; $A A$, aortic atresia; $M S$, mitral stenosis; $C F$, coronary fistula; $M A$, mitral atresia; $C A$, coronary anomalies other than coronary fistula. 
months after birth found that capillarization (as measured by diffusion distance) in both the RV and LV was reduced compared with normal hearts. They hypothesized that increased diffusion distances could affect ventricular development inasmuch as tissue aerobic metabolism is dependent on diffusion distance from capillary beds.

Our series showed no significant differences in coronary artery medial thickness between the various subtypes of HLHS with AA, but capillary density and capillary diffusion distances were not examined.

\section{Coronary Fistula}

Occurrence of coronary fistulas in AA was reported as early as 1932 by Bellet and Gouley. ${ }^{17}$ Raghib and colleagues ${ }^{18}$ described a case of AA with premature closure of the foramen ovale, with egress of LV blood through sinusoids and coronary fistulas and ultimately via the coronary sinus to the right atrium. They compared this with RV-coronary fistulas seen with pulmonary atresia and intact ventricular septum. More recently, LV-coronary fistulas in HLHS have been successfully diagnosed on prenatal echoes. ${ }^{19}$ Extensive ventricular-coronary connections to both coronary artery systems was recently reported by Roberson and his group. ${ }^{20}$ Their patient underwent hybrid stage I palliation and died at 5 weeks of age of ischemia-induced ventricular tachyarrhythmia. The left circumflex system had a proximal obstruction and was exclusively supplied through fistulous communication from the LV.

In our series we had only 1 heart with AA/MS with demonstrable coronary ostial occlusion on cardiac catheterization and coronaries filling entirely from the hypertensive LV via fistulous communication. Although 2 of the hearts in our AA/MA subgroup had coronary ostial atresia or stenosis, it is interesting to note that neither had coronary fistula.

Sathanandam and coworkers, ${ }^{21}$ in a 4-year prospective study, reported $15 \%$ incidence of ventricular-coronary connections in 100 consecutive HLHS cases, with $56 \%$ of the AA/MS subtype having ventricular-coronary connections. Mortality was limited to those cases with extensive ventricular-coronary connections. Recent work by Scheurer and colleagues $^{22}$ at our institution retrospectively reviewed all cases of AA/MS over a 42-month period beginning in January of 2007 with a significantly lower transplant-free survival $(34 \%)$ in those with LV-coronary fistulas compared with $100 \%$ in those without fistulas. In our series, AA/ MS hearts with ventricular-coronary fistulas were disproportionately represented among the specimens in the AA/ MS subgroup after palliation. This finding suggests that the presence of $\mathrm{LV}$-coronary fistulas may have contributed to mortality in the AA/MS subgroup in our series. Appendix Table 1 outlines the percentage of coronary fistulas in AA/ MS group and coronary abnormalities in the AA/MA group distributed over 10-year periods. Appendix Table 2 outlines the distribution in the pre-Norwood and post-Norwood era. As depicted in the tables, the occurrence of coronary abnormalities in autopsies in the AA/MA group is essentially unchanged over time, but the occurrence of coronary fistulas in autopsies and explanted hearts in the AA/MS group has gradually increased from $12.5 \%$ in the preoperative/preNorwood era to nearly $60 \%$ in the current era. However, because this is an autopsy series and the true denominator is unknown, we can only speculate that occurrence of coronary fistulas in the AA/MS group confers a higher risk of mortality.

The mechanism by which these ventricular-coronary connections/fistulas could contribute to an increased mortality risk remains unclear, particularly in patients after stage I palliative procedures. In contrast to patients with pulmonary atresia and RV-dependent coronary circulation, in whom proximal coronary stenosis or atresia is associated with higher mortality, ${ }^{23}$ patients with AA/MS and ventricular-coronary fistulas did not demonstrate proximal obstructions. Inasmuch as there was a high incidence of restrictive atrial septum in these patients with coronary fistulas, it is tempting to speculate that the atrial septectomy that is carried out as part of a typical Norwood or Sano stage I palliative procedure alters LV filling and thus coronary perfusion through ventricular-coronary fistulas. In addition, it is known that the AA/MS subgroup generally has a hypertensive LV with suprasytemic pressures, which may lead to reversal of flow in coronaries and coronary malperfusion. This high pressure in the LV cavity may also lead to subendocardial ischemia and fibrosis because of abnormal transmural gradients. ${ }^{24}$

\section{Limitations}

This study has limitations in that it is an autopsy series, data were collected retrospectively from a single center, and physiologic relationship between coronary fistula and mortality cannot be clearly established.

\section{CONCLUSIONS}

Coronary fistulas appear to be the most common coronary abnormality in this autopsy series of AA in HLHS. Ventricular-coronary fistulas are limited to the AA/MS group. Patients with AA/MS and coronary fistulas were overrepresented in the autopsies from the postoperative era compared with the preoperative era. The exact relationship between abnormal coronaries, particularly LV-coronary fistulas, and mortality cannot be conclusively established, but it is seems likely that coronary fistulas may have contributed to coronary ischemia and mortality in this series. Further investigation of this link between LV-coronary fistula and mortality is required.

The presence of coronary fistula on preoperative imaging studies should raise awareness of potentially increased mortality risk, and optimal management strategies need to be 
developed. The roles of preferential use of a Sano shunt for stage I palliation or a hybrid stage I palliation vs primary transplantation for neonates with significant LV-coronary fistulas should be considered in future management of patients with MS/AA and coronary fistula. Follow-up study on survivors with coronary fistulas in HLHS will help us gain a better understanding of the natural history of the coronary fistula inasmuch as there has been a recent case report of regression of fistula. ${ }^{25}$

We acknowledge Drs Stella and Richard Van Praagh since a significant portion of these data were obtained from the Cardiac Registry that they established and maintained. Their meticulous clinical notes and autopsy reports provided invaluable information.

\section{References}

1. Vida VL, Bacha EA, Larrazabal A, Gauvreau K, Dorfman AL, Marx G, et al. Surgical outcomes for patients with the mitral stenosis-aortic atresia variant of hypoplastic left heart syndrome. J Thorac Cardiovasc Surg. 2008;135:339-46.

2. Glatz JA, Fedderly RT, Ghanayem N, Tweddell JS. Impact of mitral stenosis and aortic atresia on survival in hypoplastic left heart syndrome. Ann Thorac Surg. 2008;85:2057-62.

3. O'Connor WN, Cash JB, Cottrill CM, Johnson GL, Noonan JA. Ventriculocoronary connections in hypoplastic left hearts: an autopsy microscopic study. Circulation. 1982;66:1078-86.

4. Baffa JM, Chen S-L, Guttenberg ME, Norwood WI, Weinberg PM. Coronary artery abnormalities and right ventricular histology in hypoplastic left heart syndrome. J Am Coll Cardiol. 1992;20:350-8.

5. Saroli T, Gelehrter S, Gomez-Fifer CA, van der Velde ME, Bove EL, Ensing GJ. Anomalies of left coronary artery origin affecting surgical repair of hypoplastic left heart syndrome and Shone complex. Echocardiography. 2008;25:727-31.

6. Gehrmann J, Krasemann T, Kehl HG, Vogt J. Hypoplastic left heart syndrome: the first description of the pathophysiology in 1851; translation of a publication by Dr. Bardeleben from Giessen, Germany. Chest. 2001;120:1368-71.

7. Norwood WI, Lang P, Castaneda AR, Campbell DN. Experience with operations for hypoplastic left heart syndrome. J Thorac Cardiovasc Surg. 1981;82:511-9.

8. Mahle WT, Spray TL, Wernovsky G, Gaynor WJ, Clark BJ III. Survival after reconstructive surgery for hypoplastic left heart syndrome. Circulation. 2000; 102(suppl III):III136-41.

9. Van Praagh R, Shinpo H, Kreutzer J, Geva T, Santini F. Anomalies of the mitral and aortic valves: pathologic anatomy in 659 postmortem cases. In: Pediatric Cardiology: Proceedings of the III World Congress of Pediatric Cardiology. Amsterdam: Excerpta Medica; 1990. p. 135-40.
10. Bartram U, Grunenfelder J, Van Praagh R. Causes of death after modified Norwood procedure: a study of 122 postmortem cases. Ann Thorac Surg. 1997;64: 1795-802.

11. Bharati S, Lev M. The surgical anatomy of hypoplasia of aortic tract complex. $J$ Thorac Cardiovasc Surg. 1984;88:97-101.

12. Lloyd TR, Evans TC, Marvin WJ Jr. Morphological determinants of coronary blood flow in the hypoplastic left heart syndrome. Am Heart J. 1986;112:666-71.

13. Lloyd TR, Marvin WJ Jr. Age at death in the hypoplastic left heart syndrome: multivariate analysis and importance of the coronary arteries. Am Heart J. 1989; 117:1337-43.

14. Sauer U, Gittenberger-de Groot AC, Geishauser M, Babic R, Buhlmeyer K. Coronary arteries in hypoplastic left heart syndrome. Histopathologic and histometrical studies and implications for surgery. Circulation. 1989;80(suppl I):I168-76.

15. Sugiyama H, Yutani C, Lida K, Arakaki Y, Yamada O, Kamiya T. The relationship between right ventricular function and left ventricular morphology in hypoplastic left heart syndrome: angiographic and pathological studies. Pediatr Cardiol. 1999;20:422-7.

16. Salih C, Sheppard MN, Ho SW. Morphometry of coronary capillaries in hypoplastic left heart syndrome. Ann Thorac Surg. 2004;77:903-7.

17. Bellet S, Gouley BA. Congenital heart disease with multiple cardiac anomalies. Report of a case showing aortic atresia, fibrous scar in the myocardium and embryonal sinusoidal remains. Am J Med Sci. 1932;183:458-64.

18. Raghib G, Bloemendaal RD, Kanjuh VI, Edwards JE. Aortic atresia and premature closure of foramen ovale. Myocardial sinusoids and coronary arteriovenous fistula serving as outflow channel. Am Heart J. 1965;70:476-80.

19. Patel CR, Lane JR, Spector ML, Smith PC, Waight DJ. Prenatal diagnosis of ventriculocoronary arterial communication in fetuses with hypoplastic left heart syndrome. J Ultrasound Med. 2006;25:245-9.

20. Roberson DA, Wei C, Cuneo BF, Van Bergen AH, Javois AJ, Bharati S. Extensive left ventricular to coronary artery connections in hypoplastic left heart syndrome. Echocardiography. 2008;25:529-33.

21. Sathanandam S, Cui W, Nguyen NV, Husayni TS, Van Bergen AH, Sajan I, et al. Ventriculocoronary artery connections with hypoplastic left heart: a 4 year prospective study -incidence, echocardiographic and clinical features. Pediatr Cardiol. 2010;31:1176-85.

22. Scheurer MA, Porras D, Rathod R, Emani S, Pigula FA, Lock JE, et al. Left ventricular coronary cameral fistula in the mitral stenosis aortic atresia variant of the hypoplastic left heart syndrome are associated with poor outcomes. American Heart Association Scientific Session. 2010 Nov 16. APS 310.01.

23. Giglia TM, Mandell VS, Connor AR, Mayer JE Jr, Lock JE. Diagnosis and management of right ventricle-dependent coronary circulation in pulmonary atresia with intact ventricular septum. Circulation. 1992;86:1516-28.

24. Alqranati D, Kassab GS, Lanir Y. Why is the subendocardium more vulnerable to ischemia? A new paradigm. Am J Physiol Heart Circ Physiol. 2011;300: H1090-100.

25. Ono M, Goerler H, Bertram H, Breymann T. Regression of marked myocardial sinusoids associated with hypoplastic left heart syndrome during staged Fontan approach. Ann Thorac Surg. 2010;90:1007-9.

APPENDIX TABLE 1. Distribution of various groups based on era

\begin{tabular}{|c|c|c|c|c|c|c|}
\hline Era & AA/MS total & AA/MS/CF & AA/MS/CF ( $\%$ of total) & AA/MA total & AA/MA/CA & AA/MA/CA ( $\%$ of total $)$ \\
\hline Before 1980 & 48 & 6 & $12.5 \%$ & 14 & 2 & $14 \%$ \\
\hline 1981-1990 & 47 & 10 & $21 \%$ & 38 & 3 & $8 \%$ \\
\hline $1991-2000$ & 29 & 17 & $57 \%$ & 19 & 3 & $16 \%$ \\
\hline 2001-2009 & 10 & 6 & $60 \%$ & 11 & 2 & $17 \%$ \\
\hline
\end{tabular}

$A A$, Aortic atresia; $M S$, mitral stenosis; $C F$, coronary fistula; $M A$, mitral atresia; $C A$, coronary anomalies other than coronary fistula.

APPENDIX TABLE 2. Distribution of various groups based on pre-Norwood (before 1980) and post-Norwood (after 1980) era

\begin{tabular}{|c|c|c|c|c|c|c|c|}
\hline Era & AA/MS total & AA/MS/CF & AA/MS/CF ( $\%$ of total) & AA/MA total & AA/MA/CA & AA/MA/CA ( $\%$ of total) & $P$ value \\
\hline Before 1980 & 48 & 6 & $12.5 \%$ & 14 & 2 & $14 \%$ & 1.0 \\
\hline After 1980 & 86 & 33 & $38 \%$ & 68 & 8 & $12 \%$ & .002 \\
\hline$P$ value & & & .0015 & & & 1.0 & \\
\hline
\end{tabular}

$A A$, Aortic atresia; $M S$, mitral stenosis; $C F$, coronary fistula; $M A$, mitral atresia; $C A$, coronary anomalies other than coronary fistula. 